\title{
DIDÁCTICA DE LAS ARTES CON TIC, APRENDIZAJE COLABORATIVO E INTERDISCIPLINARIEDAD EN LA FORMACIÓN INICIAL DEL PROFESORADO
}

\author{
DIDACTIC OF THE ARTS WITH ICT, COLLABORATIVE LEARNING AND INTERDISCIPLINARITY \\ IN THE INITIAL TRAINING OF TEACHERS \\ DIDÁTICA DAS ARTES COM TIC, APRENDIZAGEM COLABORATIVA E INTERDISCIPLINARIDADE \\ NA FORMAÇÃO INICIAL DE PROFESSORES
}

\author{
${ }^{1}$ Universidad de Valencia, España, Rosa.Isusi@uv.es \\ 2Universidad de Valencia, España, Elena.Saez@uv.es \\ ${ }^{3}$ Universidad de Valencia, España, jorge.lizandra@uv.es
}

Rosa Isusi-Fagoaga ${ }^{1}$, Elena Sáez Alonso ${ }^{2}$, Jorge Lizandra ${ }^{3}$

\section{RESUMEN}

El aprendizaje mediante las tecnologías de la información y comunicación y la interdisciplinariedad como ejes de la innovación pedagógica son aspectos que se deben fomentar en la Educación Superior desde la declaración de Bolonia, hace ya veinte años. Sin embargo, todavía no han arraigado lo suficiente en muchas aulas universitarias. Es por ello que se presenta un estudio sobre una experiencia educativa desarrollada por profesorado de las áreas de didáctica de la expresión musical, plástica y corporal con futuros docentes en formación. Los objetivos son promover el uso de las TIC, nuevos sistemas de comunicación de los trabajos académicos y el aprendizaje colaborativo e interdisciplinar entre los estudiantes. Todo ello contribuye a una renovación tanto en la metodología docente como en el proceso de evaluación, otorgando un papel más activo al alumnado a la vez que favoreciendo la comunicación y coordinación entre el profesorado. La metodología empleada ha sido fundamentalmente cualitativa y el método la investigación-acción. Las técnicas de recogida de datos han sido cuestionarios realizados al alumnado, el diálogo interactivo, cuaderno de campo del profesorado y la evaluación grupal de los trabajos realizados con TIC del alumnado. La muestra han sido 140 estudiantes del Grado de Maestro en Educación Infantil de la Universitat de València. Los resultados muestran la satisfacción del alumnado y profesorado a lo largo de todo el proceso. Se puede concluir que la experiencia y estudio muestra una buena práctica innovadora que puede ser optimizada en el futuro y/o servir de inspiración a otros docentes universitarios.

Palabras Claves: innovación pedagógica; educación artística; evaluación; formación de profesorado; TIC.

\begin{abstract}
Learning through information and communication technologies and interdisciplinarity as axes of pedagogical innovation, are aspects that should be promoted in Higher Education since the Bologna declaration, twenty years ago. However, they have not yet taken root enough in many university classrooms. That is why a study on an interdisciplinary experience developed by teachers in the didactic areas of musical, artistic and body expression with future teachers in training is presented. The objectives are to promote the use of ICT, new communication systems for academic work and collaborative and interdisciplinary learning among students. All this contributes to
\end{abstract}

52 Didáctica de las artes con Tic, aprendizaje colaborativo e interdisciplinariedad en la formación inicial del profesorado 
a renewal both in the teaching methodology and in the evaluation process, giving a more active role to the students while stimulating communication and coordination among the teaching staff. The methodology used has been fundamentally qualitative and the research-action method. The data collection techniques have been questionnaires for the students, the interactive dialogue, the teachers 'field notebook and the group evaluation of the students' audio-visual works. The sample was made up of 140 students from the Master's Degree in Early Childhood Education at the University of Valencia. The results show the satisfaction of the students and teachers throughout the entire process. It can be concluded that the experience and study show an innovative good practice that can be optimized in the future and / or serve as inspiration for other university teachers.

Key words: pedagogical innovation; art education; assessment; teacher training; ICT

\section{RESUMO}

A aprendizagem pelas tecnologias de informação e comunicação e a interdisciplinaridade como eixos de inovação pedagógica são aspetos que devem ser promovidos no Ensino Superior desde a declaração de Bolonha, há vinte anos. No entanto, eles ainda não estão arraigados o suficiente em muitas salas de aula da faculdade. Para isso é apresentado um estudo sobre uma experiência educacional desenvolvida por professores nas áreas didáticas de expressão musical, plástica e corporal com futuros professores em formação. Os objetivos são promover o uso das TIC, novos sistemas de comunicação para o trabalho acadêmico e a aprendizagem colaborativa e interdisciplinar entre os alunos. Tudo isto contribui para uma renovação tanto na metodologia de ensino como no processo de avaliação, dando um papel mais ativo aos alunos e favorecendo a comunicação e coordenação entre o corpo docente. A metodologia utilizada foi fundamentalmente qualitativa e o método da pesquisa-ação. As técnicas de coleta de dados foram: questionários aplicados aos alunos, diálogo interativo, caderno de campo dos professores e avaliação em grupo dos trabalhos realizados com as TIC pelos alunos. A amostra foi de 140 alunos do Mestrado em Educação Infantil da Universidade de Valência. Os resultados mostram a satisfação dos alunos e professores ao longo de todo o processo. Pode-se concluir que a experiência e o estudo mostram uma boa prática inovadora que pode ser otimizada no futuro e / ou servir de inspiração para outros professores universitários.

Palavras chaves: inovação pedagógica; educação artística; avaliação; formação de professores; TIC.

\section{1.- INTRODUCCION}

El proceso de Convergencia al Espacio Europeo de Educación Superior (EEES) tiene entre sus objetivos la mejora de la calidad de la enseñanza universitaria y para ello se precisan de experiencias de innovación educativa que ayuden a una mayor cualificación de los estudiantes durante su formación inicial como futuros docentes y también como futuros ciudadanos (CollSerrano et al., 2018).

El modelo formativo tradicional de la enseñanza universitaria se ha venido caracterizando por el predo- minio de las clases magistrales basadas en la comunicación oral, una fragmentación del conocimiento, un contenido curricular organizado y secuenciado de forma cerrada, una desconexión entre las enseñanzas teóricas y prácticas, una escasa comunicación y colaboración entre el profesorado, así como un sistema de evaluación finalista sobre los resultados (adquisición de conocimientos) de los estudiantes (Lavega et al., 2013). Sin embargo, desde la constitución y desarrollo del EEES, se ha recomendado métodos de aprendizaje interdisciplinar y colaborativo con uso de herramientas propias de las tecnologías de la información y co- 
municación (TIC) que respondan mejor a las actuales demandas sociales (Pareja y Pedrosa, 2014) y que favorezcan la innovación cuando se demuestra que se han aplicado con éxito (Castro-Martínez y Fernández de Lucio, 2013).

Es innegable que las TIC, desarrolladas fundamentalmente desde los años 1990 del siglo pasado para una gestión de la información y de la comunicación más eficiente, han cambiado tanto el acceso a la información como el comportamiento humano. Ello se ha visto robustecido por el invento y acceso a Internet y el desarrollo de nuevos dispositivos de tecnología digital. Las TIC facilitan el acceso a una gran cantidad de información, favorecen la conexión entre instituciones y la comunicación entre personas, contribuyen a la creación colectiva del conocimiento transformación y promueven nuevas formas de pensamiento. Al facilitar el acceso a una cantidad ingente de conocimiento, es necesario potenciar el espíritu crítico para poder seleccionar y gestionar adecuadamente el conocimiento. Todo ello son aprendizajes que se deben promover entre los estudiantes universitarios y especialmente entre los que serán futuros docentes. Para aprovechar de manera efectiva el poder de las TIC en Educación, han de darse una serie de condiciones recomendadas por la UNESCO: a) Los estudiantes y docentes han de tener acceso a las tecnologías e Internet en las clases; b) ambos deben tener acceso a contenidos educativos en formato digital; y c) los docentes deben tener los conocimientos y habilidades necesarias para ayudar a los estudiantes a alcanzar los objetivos académicos (Khvilon y Patru, 2004). Sin embargo, existen algunas barreras que han dificultado la amplia difusión y utilización de las TIC en el sistema educativo. Las más significativas son la falta de dispositivos y de software, los problemas técnicos y de tiempo derivados del aprendizaje y uso de los mismos y, sobre todo, la falta de formación del profesorado, su desconfianza hacia estos dispositivos y su resistencia al cambio y a la innovación (Schoepp, 2005). Muchas de estas barreras todavía siguen existiendo y eliminarlas requiere de una acción gradual co- lectiva por parte de las instituciones y del profesorado (Drent y Meelissen, 2008).

Las TIC a su vez favorecen los métodos de aprendizaje colaborativo, y estos a su vez la comunicación entre pares. Ambos, permiten conectar e implicar a los estudiantes en su propio proceso de enseñanza-aprendizaje a través de grupos pequeños y heterogéneos en el marco de un planteamiento que potencie la interacción y el aprendizaje dialógico entre ellos (Aubert et al., 2008). En este proceso es imprescindible que el docente adquiera la función de guía-mediador que asegure la participación igualitaria y fomente el trabajo en equipo, en el que cada estudiante comprende que su trabajo beneficia a los compañeros y toda la información que llega al grupo debe de incorporarse en el mismo para ser utilizada y que el conjunto del grupo tenga éxito en la tarea (Sánchez-Valero, Arrazola-Carballo y CalderónGarrido, 2017). Esto implica que el aprendizaje colaborativo precisa de una intencionalidad explícita por parte de todos los participantes y aunque son múltiples las investigaciones que señalan las ventajas del empleo de estructuras de aprendizaje colaborativo, estos procesos no están exentos de dificultades, especialmente en la universidad, debido a que, unas veces por la gran cantidad de alumnado, otras por complejidad de la coordinación en las tareas del profesorado, es frecuente resistirse a dichos cambios (Fullam, 2002; Vallés-Rapp, Gil-Puente y López-Luengo,2019). De ahí la relevancia de rodearse de profesionales que entiendan que los procesos de aprendizaje, tradicionalmente basados en la trasmisión magistral de contenidos, deben ir dejando paso a una metodología basada en el trabajo activo de todos aquellos que forman parte del mismo (Castillo-Cedeño, Ramírez-Abrahams y Ruíz-Guevara, 2017). Paralelamente, si se favorecen los procesos de enseñanza-aprendizaje colaborativo consecuentemente el proceso de evaluación debe ser coherente y por tanto debe incluir la evaluación grupal.

Asimismo, incentivando no solamente el aprendizaje colaborativo sino también un aprendizaje integrado, 
cada vez más se promueve la intervención de los educadores como facilitadores de un aprendizaje donde los contenidos formativos se lleven a cabo de una forma global, integrando unos y otros (Summers, Childs y Corney, 2005). Esto implica que las propuestas educativas tienen que presentar de una forma clara la relación entre los aprendizajes que interesan más directamente a los estudiantes para llegar a conseguir los objetivos que se pretenden, no solamente la inserción laboral sino también la emancipación de los estudiantes y una participación ciudadana más activa. Surge así la interdisciplinariedad como una propuesta metodológica que da respuesta a la necesidad de presentar los contenidos de aprendizaje de forma interrelacionada en la formación universitaria.

En este contexto, cada vez más el aprendizaje colaborativo y la interdisciplinariedad, que supone una coordinación del profesorado, son considerados como esenciales para la Educación Superior actual (Lavega et al. 2013; Rivera y De la Torre, 2006). No obstante, es poco frecuente encontrar literatura que aborde estas cuestiones desde el ámbito de la educación, y menos frecuente todavía aunando las áreas de música, expresión corporal y artes visuales, a pesar que frecuentemente forman parte del mismo departamento en las facultades de formación del profesorado en las universidades españolas (Isusi-Fagoaga, 2018; Martínez-Bello y Bernabé-Villodre, 2019).

Existen trabajos que tratan de abordar el aprendizaje integral e interdisciplinar a través de la música, plástica (grafismo) y educación física o corporal (movimiento, psicomotricidad) basado en talleres multidisciplinares con una amplia tradición en la Educación Infantil (Vila y Cardo, 2009). También existen varias experiencias e investigaciones sobre renovación de metodologías docentes y herramientas tecnológicas centradas fundamentalmente en el ámbito artístico-musical de la Educación Secundaria (Botella y Adell, 2018). Asimismo, en relación con la formación universitaria, existe una experiencia realizada sobre interdisciplinariedad artística universitaria en el ámbito de la formación de maestros (Mínguez et al., 2014). Del mismo modo, aunque la educación artística se caracteriza por ser una disciplina que ha tardado en incorporarse al ámbito de las ciencias sociales, comienza a ser cada vez más frecuente encontrar trabajos sobre la investigación al respecto (Díaz y Giráldez, 2013).

\section{2.- OBJETIVOS}

Los principales objetivos son, por una parte, promover el uso de las TIC, nuevos sistemas de comunicación de los trabajos académicos y el aprendizaje colaborativo e interdisciplinar entre los estudiantes y, por otra, proponer y comunicar experiencias que han sido aplicadas con éxito y que contribuyen a renovar el modelo educativo anterior.

\section{3.- METODOLOGÍA}

Para alcanzar los objetivos se ha optado por una metodología fundamentalmente cualitativa y en concreto se ha seguido el método de espirales de investigaciónacción. Este permite analizar, en un proceso en espiral, los estadios de planificación, acción y evaluación de los resultados de una determinada acción. Autores pioneros como Carr y Kemmis (1988) destacaron la importancia de este modelo que une los aspectos teóricos con los prácticos en la enseñanza. Consideraban que, el profesorado debía proponer y necesita asumir la investigación educativa para no ser un mero ejecutor, sino para ser capaz de imaginar nuevas formas de enseñanza que permitan mejorar los logros y su práctica en el aula, así los docentes se convertían en sujetos críticos e investigadores de su propia realidad. En concreto, para nuestro trabajo, como docentes de la asignatura Estimulación e intervención temprana: música, grafismo y movimiento (objeto de análisis en el presente estudio), hemos observado nuestra práctica docente, la hemos analizado y evaluado, con el fin de ofrecer una primera propuesta de innovación educativa en la promoción 
del aprendizaje colaborativo e interdisciplinar.

Con esto, lo que se ha pretendido es desarrollar una investigación educativa, mediante la que se busca actuar directamente sobre la docencia con la intención de mejorarla. Por tanto, la investigación educativa requiere de un proceso de indagación desde dentro del contexto, por lo que tanto el alumnado como el propio profesorado son considerados como participantes activos del proceso de investigación (Blández, 2010). Esto significa que en cierta medida los agentes del proceso de enseñanza-aprendizaje en el que nos encontramos inmersos necesariamente, actuamos como instrumentos que filtran la información obtenida, a la vez que aportan aquella que se considera necesaria para una mejor comprensión del discurso (Gil, Rodríguez y García 1996; Martínez, 2014).

\section{1.- Muestra}

La experiencia que presentamos ha tenido lugar en el Grado de Maestro/a en Educación Infantil de la Universitat de València, concretamente con tres grupos de aproximadamente 45 estudiantes cada uno de la asignatura de Estimulación e intervención temprana: música, grafismo y movimiento. Esta asignatura es cuatrimestral y cuenta con 60 horas de formación presencial (6 créditos ECTS), divididos en tres secciones de 20 horas, destinadas al desarrollo de cada una de las áreas de conocimiento de didáctica de la expresión musical, plástica y corporal. El estudio se llevó a cabo durante el segundo cuatrimestre del curso 2016/17 en el que habían matriculados un total de 140 estudiantes de edades comprendidas entre los 20 y los 28 años.

\section{2.- Diseño}

En los meses previos al inicio de la asignatura, el equipo docente se reunió para conformar la planificación y el método de trabajo que se llevaría a cabo a lo largo del período lectivo. Los objetivos fundamentales de la metodología de trabajo fueron facilitar la enseñanza de los contenidos de la asignatura desde la interdisciplinariedad en la educación artística universitaria, así como el trabajo en grupo y colaborativo con el resto de compañeros de clase. Además, promover el uso de las TIC durante el proceso y presentación de trabajos y así contribuir a que pudiera servir de orientación a los docentes que estén impartiendo esta u otra asignatura interdisciplinar.

Fruto del cometido de comunicación y coordinación docente, se redactó un documento de apoyo en el que se plasmaron tanto las directrices generales en cuanto a temporalización, la metodología y criterios de evaluación. Este documento se compartió al inicio del curso con el alumnado en el aula virtual, con la intención de ser una guía para llevar a la práctica el método de innovación pedagógica pensado para ese curso. Tomando como referencia las propuestas didácticas de Vila y Cardo (2009) de la estimulación de la curiosidad del alumnado con el fin de despertar el aprendizaje de nuevos conocimientos, cada docente en sus clases proporcionó al alumnado materiales tanto en formato papel como digital y recursos interdisciplinares para la adquisición de contenidos. Asimismo, se proporcionó a los estudiantes recursos didácticos y fuentes documentales específicas de cada una de las áreas como, por ejemplo, en el caso de didáctica de la música se realizaron reflexiones críticas en grupo sobre una selección de textos sobre la importancia de la música en la Educación Infantil y sobre un tema controvertido como es el de las aptitudes musicales innatas (Akoschky et al., 2008).

Posteriormente los alumnos comenzaron a elaborar el trabajo final interdisciplinar, que consistió en la creación de una propuesta didáctica para Educación Infantil centrada en la estimulación temprana a partir de espacios multisensoriales en la que se integrarían los aprendizajes de las áreas de didáctica de la expresión musical, plástica y corporal. Para ello, cada grupo de alumnos (7 grupos en cada una de las áreas de estu- 
dio de la asignatura - música, grafismo y movimiento, en total 21 grupos). Este trabajo debía elaborarse mediante las TIC y presentarlo en formato audiovisual y/o grabarse en vídeo (3-5 minutos), para ser expuesto presencialmente en las últimas sesiones conjuntas de la asignatura. Esto suponía una novedad con respecto a la tradición anterior en la que el trabajo se presentaba escrito en papel. El material audiovisual tenía que aportar unos contenidos mínimos como: una breve justificación del trabajo final con información relacionada con el contexto en el que se tenía previsto desarrollar, así como la explicación de los objetivos y finalidades de cada actividad, junto con algún tipo de ejemplificación visual de las actividades a realizar. Asimismo, los alumnos plasmaron una valoración crítica del trabajo realizado y las observaciones que se consideraran pertinentes. Este trabajo final supuso un 25\% del total de la nota global de la asignatura, teniendo como criterios de evaluación básicos el disponer de una capacidad de comunicar las ideas con claridad, que en las actividades se interrelacionaran contenidos de las tres áreas de conocimiento y que se adecuaran a los contenidos trabajados en el desarrollo de la asignatura, el uso adecuado de los recursos tecnológicos, la asistencia a las sesiones previas de preparación de la exposición y la adecuación al tiempo del que disponían para realizar la exposición (entre 8-10 minutos). El resto de la nota correspondía $25 \%$ a cada una de las tres áreas implicadas (didáctica de la música, plástica y corporal).

Al final del desarrollo de las clases, se propuso una actividad grupal de evaluación consistente en que cada grupo dedicara un tiempo para reflexionar sobre la experiencia vivida en la asignatura, tras la que redactarían un informe de evaluación.

\section{3.- Técnicas de recogida de datos}

Las técnicas de recogida de datos y fuentes de información de este estudio se obtuvieron a partir de: (i) cuestionarios escritos a los estudiantes a través del aula virtual (Moodle) de la universidad, (ii) el diálogo interactivo entre docentes y estudiantes, (iii) la observación y el cuaderno de campo realizado del profesorado, (iv) la evaluación de los trabajos realizados por los estudiantes; (v) y la confección de un trabajo de exposición de los estudiantes en formato audiovisual.

Cada grupo de estudiantes tuvo que consensuar las respuestas al cuestionario y contestaron de forma grupal. En el informe de evaluación de los proyectos presentados se propuso realizar un documento en el que se plasmaran las reflexiones al respecto. Para orientar a los alumnos se les propuso unas preguntas a las que podían responder, con las que se trataba de recopilar los elementos clave de la experiencia (véase Tabla I). Estas preguntas eran de carácter abierto y responderlas era una tarea totalmente voluntaria, que no tenía asociada ningún porcentaje dentro de la evaluación final de la asignatura. Así pues, de los 21 grupos de trabajo organizados entre los alumnos (7 por cada grupo de la asignatura), se obtuvo respuesta de 10 , lo que supone un $47,7 \%$ del total de subgrupos.

Las preguntas orientativas para la actividad de evaluación de la experiencia por parte de los estudiantes han sido:

A) Indica aspectos positivos y/o negativos de haber recibido una asignatura en la que se han desarrollado contenidos de expresión musical, plástica y corporal.

B) Indica aspectos positivos y/o negativos del trabajo final con TIC (espacio multisensorial)

C) Indica aspectos positivos y /o negativos de haber unido en la exposición de los trabajos a los tres grupos con los que se ha desarrollado la asignatura.

\section{4.- Procedimiento de análisis}

La aproximación metodológica cualitativa adoptada en este estudio implica el desarrollo de un análisis de los datos que preserve la naturaleza textual de los mismos. Por otro lado, las respuestas del alumnado pueden es- 
tar relacionadas con los aspectos de interés del equipo investigador o con otros aspectos relevantes para los informantes y que son por consiguiente susceptibles de análisis. Así pues, el análisis consistió en identificar unidades textuales de las respuestas de los estudiantes y dotarlas de sentido conjunto agrupándolas y clasificándolas en unidades temáticas o categorías, a través de un proceso de categorización inductivo, tal y como se indica en la literatura especializada en este tipo de análisis (Rodríguez, Gil y García, 1996). Es decir, para el establecimiento de las categorías (unidades temáticas o datos) se tuvo en cuenta tanto la frecuencia de aparición del dato, la calidad de la información o la relevancia de la aportación. Dado el objetivo de este estudio y el interés del equipo investigador, el propósito se centró en la obtención de las categorías relacionadas con el trabajo interdisciplinar, el aprendizaje colaborativo y la metodología innovadora. Asimismo, dado el carácter crítico y constructivo de esta propuesta de innovación docente, se decidió añadir una categoría de aspectos de mejora que invitara a la reflexión y revisión de nuestra propuesta, con el fin de poder mejorarla en el futuro. La Figura 1 muestra estas cuatro categorías principales. Sin embargo, de la interpretación de las unidades temáticas surgieron otras subcategorías anidadas a las principales, tal como explicamos a continuación en el apartado de resultados y discusión.

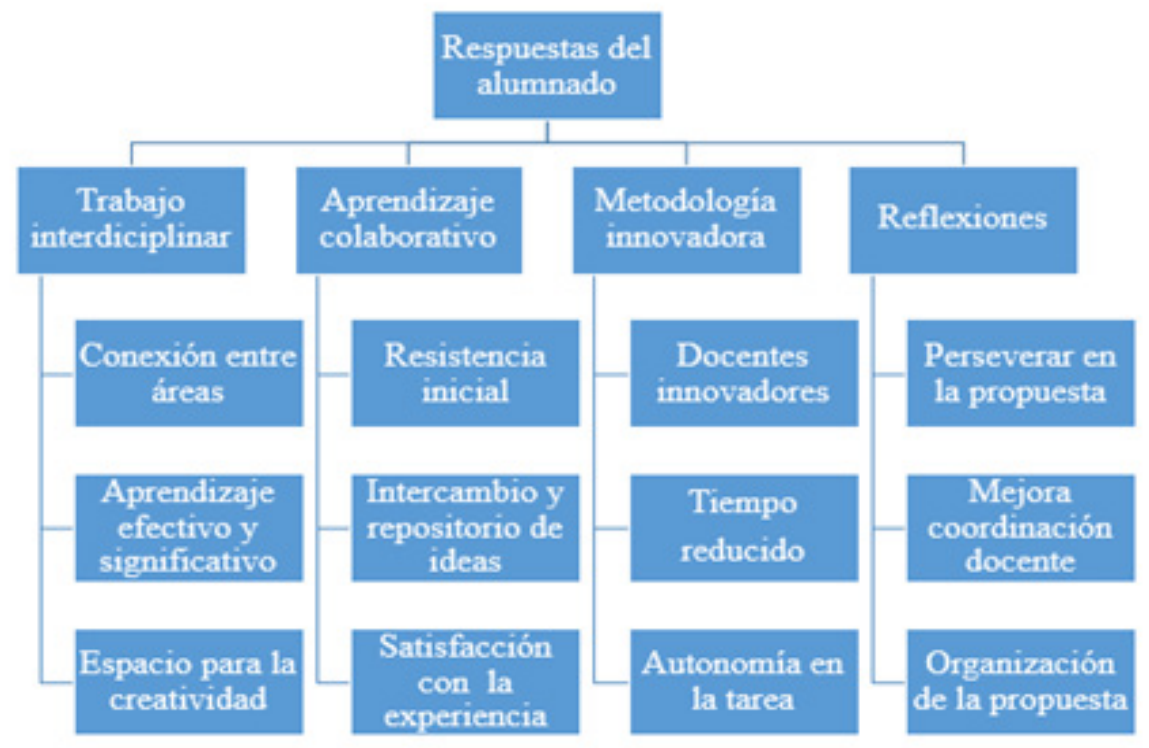

Figura 1. Categorías y subcategorías resultantes del análisis cualitativo.

Fuente: Elaboración propia.

\section{4.- RESULTADOS Y DISCUSIÓN}

El apartado de resultados y discusión se ha organizado a partir de las categorías emergidas en el análisis de los datos, estableciéndose de la siguiente manera:

\section{1.- Trabajo interdisciplinar}

El alumnado consideró útil el enfoque interdisciplinar de la asignatura, pues les permitió interconectar y practicar contenidos de las tres áreas de conocimiento de didáctica de la expresión musical, plástica y corpo- 
ral. Un ejemplo explícito de ello fue la realización del trabajo final de creación de espacios multisensoriales, que según palabras de una de las estudiantes: "me ha permitido hacer una recopilación de las tres áreas de conocimiento que hemos ido trabajando durante las clases y, por tanto, mejorar estos aprendizajes" (Grupo de estudiantes 1 ).

El hecho de abordar la estimulación e intervención temprana desde tres prismas diferentes y complementarios, supuso un esfuerzo de asimilación e integración de los contenidos por parte del alumnado. Con el esfuerzo realizado se logró que profundizaran y ampliaran el conocimiento, tanto de las propias áreas como de las posibilidades de interacción entre las mismas:

Haber trabajado contenidos de las tres áreas nos ha dado la oportunidad de ampliar nuestros conocimientos de cada una de ellas, para posteriormente poder integrarlos todos en actividades poco comunes, en comparación a las que se realizan actualmente en las aulas (Grupo de estudiantes 3 ).

Haber desarrollado contenidos de las tres áreas es tener una visión más amplia sobre cada una de estas. Ya que al darla un profesor específico y en un tiempo determinado permite que te centres de lleno en el área que estás trabajando en ese momento. Permitiendo aclarar todos los conceptos y trabajar todos los aspectos de forma más sencilla (Grupo de estudiantes 5).

Consideramos que las 3 áreas se complementan a la perfección [...] La música tiene la capacidad innata de enriquecer cualquier actividad, la parte de corporal suele estar implícita de una manera u otra, y la parte de grafismo siempre está muy presente en la educación infantil (Grupo de estudiantes 4).

La experiencia de interrelacionar áreas de conocimiento permitió al alumnado aprender a establecer relaciones de ideas a través de las cuales pudieron comprobar que: "el aprendizaje es más efectivo y más significativo cuando se 'mezclan' las diferentes áreas de experiencia" (Grupo de estudiantes 6).

Al mismo tiempo, algún alumno afirmó que para lograr plantear tareas interdisciplinares fue necesario: "sacar nuestra creatividad e imaginación, al combinar actividades que ya conocemos y tener que replantearlas" (Grupo de estudiantes 10).

Estos resultados coinciden con el trabajo de Morales y Landa (2004) en el que este tipo de propuestas interdisciplinares, en el que se trata de aunar conceptos y aprendizajes de diferentes disciplinas, supone una tarea intelectual de nivel superior que facilite el desarrollo de procesos metacognitivios. Asimismo, para abordar esa tarea intelectual, el rol del docente debe ser de facilitador y guía en todo el proceso de aprendizaje, estimulando la capacidad creativa del alumnado y por tanto obteniendo un aprendizaje más significativo (Iglesias-Cortizas y Rodicio-García, 2013).

\section{2.- Aprendizaje colaborativo}

Se ha observado que para promover el logro de experiencias de aprendizaje colaborativo la constitución de pequeños grupos, entre tres y cuatro integrantes ha sido clave, al igual que el intervalo de tiempo para la elaboración de trabajos conjuntos, y el desarrollo del trabajo final en formato audiovisual. Sin embargo, conviene destacar que al informar al alumno que la metodología colaborativa conllevaba la exposición pública de sus trabajos finales ante el resto de los grupos de la asignatura, el alumnado mostró una cierta resistencia inicial del proceso a participar: "En un primer momento no nos gustó mucho la idea de tener que exponer mi trabajo delante de 3 clases, ya que pasaría bastante vergüenza" (Grupo de estudiantes 6). "Con respecto a exponer nuestros proyectos delante de los tres grupos, al principio no nos gustó la idea, ya que el grupo reducido pasaba a ser muy extenso" (Grupo de estudiantes 7). 
Sin embargo, el alumnado tras la exposición de su trabajo destacó el valor pedagógico de realizar esta actividad ya que: "una vez realizada ésta nos dimos cuenta que al poder escuchar a todos los compañeros teníamos más posibilidades de sacar nuevas ideas y ver diferentes trabajos" (Grupo de estudiantes 6).

$\mathrm{Y}$ es que esa posibilidad de compartir e intercambiar ideas fue uno de los elementos más destacados por el alumnado en esta experiencia. El hecho de organizar las sesiones conjuntas de exposiciones del trabajo interdisciplinar permitió que: "observar una gran variedad de propuestas diferentes [...] ha sido enriquecedor poder aprender de la distinta forma de pensar de nuestros compañeros, así como de los errores o las virtudes de sus proyectos" (Grupo de estudiantes 5).

Estos resultados están acordes a lo indicado por Fullam (2002), según el cual, los procesos de cambio en educación suelen partir de una posición de resistencia inicial debido a la dimensión subjetiva de aquellos que la experimentan. Esto significa que el éxito o el fracaso de las propuestas novedosas va a depender en gran medida de cómo de interesantes, útiles o necesarias las perciban los participantes, así como de otros factores interpersonales o intrapersonales. No obstante, vencida esa resistencia inicial, se observó como el trabajo colaborativo se presentó como una buena oportunidad para contribuir al aprendizaje de los demás, al mismo tiempo que se obtuvo una retroalimentación basada en el conocimiento de los compañeros, reforzando con ello la intención de lograr aprendizajes más sólidos a partir del uso de metodologías activas (Coutinho, 2007).

\section{3.- Metodología innovadora}

El alumnado destacó la predisposición del profesorado por "realizar asignaturas activas y útiles en cuanto a nuestra futura práctica docente" (Grupo de estudiantes 2). También algún estudiante destacó cómo el planteamiento de la asignatura fue más allá de los contenidos para facilitar "el desarrollo de habilidades sociales y profesionales" (Grupo de estudiantes 3).

No obstante, fueron varios los comentarios que aludieron la falta de tiempo para desarrollar los contenidos de las áreas con una mayor profundidad. En palabras de una de las informantes, ésta ha sido para su grupo "una pequeña muestra de lo que podemos realizar" (Grupo de estudiantes 3). Tanto es así que otra informante alude a la posibilidad de que la asignatura fuera "anual, ya que en muy poco tiempo se pretende dar mucho contenido y abordar temas muy diversos" (Grupo de estudiantes 4). Esta percepción de falta de tiempo se percibió también en las exposiciones del trabajo final ya que "al realizarse exposiciones una tras otra, no ha sido fácil asimilar tan bien aquello que nos mostraban los compañeros y compañeras" (Grupo de estudiantes 1).

A pesar de estas incidencias, el alumnado destacó "la libertad con la que se han podido unir estas tres áreas mediante los circuitos multisensoriales, por la que cada grupo hemos escogido cómo hacerlo" (Grupo de estudiantes 8), facilitando con ello la autonomía en el trabajo, tanto individual como grupal.

Como docentes son numerosos los elementos de los procesos de enseñanza-aprendizaje que podemos modificar (la metodología, el estilo de enseñanza, la evaluación...). No obstante, hay otros aspectos como la carga lectiva sobre los que resulta mucho más difícil intervenir. Además, en los últimos años la convergencia hacia el EEES ha tenido como consecuencia la reducción de asignaturas anuales a cuatrimestrales y con ello, en muchos casos, a la proliferación de asignaturas compartidas entre distinto profesorado. Este tipo de medidas no suponen en sí un problema, salvo en situaciones en las que se pretende llevar a cabo propuestas de trabajo innovadoras, dado que requieren de tiempo para ser comprendidas y asimiladas, especialmente cuando se desarrollan por primera vez. En ese sentido, según Kirk (1990), convendría tener presente la visión del profesorado que se inicia en procesos 
de innovación, dado que desde la experiencia práctica se detectan necesidades que difícilmente se podrían determinar desde estamentos ajenos al contexto de aprendizaje. Por otro lado, este tipo de situaciones requieren a su vez de un firme convencimiento sobre la necesidad de una buena coordinación docente, hecho que consideramos que es positivo para el buen funcionamiento de los procesos de enseñanza-aprendizaje y que a su vez permite asumir y refrendar las directrices propuestas desde los departamentos y la comisión académica de las titulaciones académicas.

\section{4.- Reflexiones del alumnado}

Como se comentó al principio de este apartado, han sido tan pertinentes las observaciones del alumnado y las propuestas de mejora, que se consideró conveniente recogerlas en un epígrafe específico.

En primer lugar, se animó al profesorado a repetir la propuesta es sucesivos cursos, manteniendo tanto las exposiciones de los trabajos con TIC juntando los tres grandes grupos participantes, como las sesiones finales destinadas a la tutorización del trabajo multisensorial.

No obstante, hubo varios comentarios en los que se hacía referencia a posibles contradicciones en las indicaciones que ofrecíamos los distintos docentes de la asignatura, que en algunos casos han hecho: "un tanto complicado realizar el trabajo teniendo en cuenta el criterio de cada uno de los maestros y maestras" (Grupo de estudiantes 1); "Mejoraría la organización a la hora de crear los grupos de trabajo" (Grupo de estudiantes 3); "Desearíamos que hubiera una organización más clara por parte de los maestros de la asignatura, ya que pensamos que algunas cosas no estaban del todo claras" (Grupo de estudiantes 7); "Pensamos que se debería mejorar la trasmisión de la información inicial para ayudar a los alumnos ante el desconocimiento inicial que este trabajo final supone" (Grupo de estudiantes 5).

Además, se sugirió “dedicar la sesión de inicio de curso a explicar la actividad en profundidad contando con la presencia de los tres profesores y que cada profesor nos diera unas pautas de lo que se valora desde cada área" (Grupo de estudiantes 10).

Finalmente, aunque se consideró conveniente mantener las exposiciones de los trabajos ante la audiencia, se propuso "realizar grupos de trabajo más pequeños, para así, realizar las exposiciones solo a una clase en la que habrá también diversidad de ideas, pero todo sería más cómodo" (Grupo de estudiantes 4)

Esta experiencia de trabajo se correspondió con las primeras fases de un proceso de innovación educativa basado en las espirales de investigación-acción (Carr y Kemmis, 1988). Esto significa que una vez desarrollada la propuesta conviene reflexionar sobre ella y formular aquellos elementos del proceso que se consideren necesarios para optimizarla. Una vez efectuados esos cambios en la propuesta, se contaría con una nueva propuesta que sería sometida al mismo proceso, logrando con cada ajuste alcanzar una propuesta de trabajo cuya finalidad no sea solamente ser mejorada, sino que a su vez genere aprendizajes más eficaces para las tareas creadas. De ahí que la contribución del alumnado, no solo en la vivencia sino en la evaluación de la experiencia, resulte fundamental, ya que para lograr auténticos procesos de investigación-acción conviene abrir y compartir la experiencia tanto a otros docentes como a los y las estudiantes (Calvo y Susinos, 2010). Con este trabajo interdisciplinar y colaborativo, no solo se pretendió generar dinámicas de aprendizaje en la que participaran todos los agentes educativos, sino que a su vez contribuyeran a la normalización de estas prácticas docentes.

\section{5- CONCLUSIONES}

Se ha observado y conseguido una satisfacción tanto entre el alumnado como en el profesorado no solo ante la utilización de las TIC y el trabajo colaborativo e interdisciplinar, sino también en el desarrollo de un 
método práctico, algo que se ha visto reflejado en el interés y la calidad técnica de los trabajos audiovisuales realizados por parte del alumnado.

Los trabajos elaborados por los estudiantes han reflejado una gran destreza técnica en el manejo de las TIC a la vez que han mostrado la interdisciplinariedad que se pretendían como objetivos relacionando de forma coherente y complementaria los conocimientos y propuestas de las tres áreas implicadas. El hecho de que el requisito del trabajo fuera su presentación en formato audiovisual, suprimiendo la versión en papel, ha facilitado tanto la tarea del alumnado, que ya habitualmente utilizaba con anterioridad de forma espontánea algún tipo de soporte con TIC (power point) como soporte para su exposición oral, como la del profesorado en la evaluación del trabajo final. Además de la competencia digital, entre otras, se han fomentado tanto temas transversales como los objetivos del desarrollo sostenible mediante el respeto al medio ambiente, al suprimir el formato papel tanto de los trabajos como de la evaluación final.

Otra de las novedades de esta experiencia ha sido la exposición de los trabajos finales del alumnado ante los tres grupos de estudiantes juntos, es decir, ante un total de 140 personas. Este hecho ha enriquecido considerablemente el proceso de enseñanza-aprendizaje y ha fomentado tanto el aprendizaje interactivo entre pares como el sentido crítico.

La realización de esta experiencia de innovación docente ha fomentado la comunicación y coordinación entre el profesorado de las tres áreas implicadas de didáctica de la expresión musical, plástica y corporal en la asignatura de Estimulación temprana: música, grafismo y movimiento, hecho que ha resultado ser no solo enriquecedor, sino además satisfactorio tanto para los docentes como un modelo para los estudiantes.

Por tanto, puede concluirse que esta propuesta que ha aglutinado aspectos como la promoción de las TIC y el aprendizaje colaborativo e interdisciplinar ha sido una buena experiencia que ha mejorado la práctica docente de la asignatura implicada. Además, ha contribuido a una renovación tanto en la metodología docente como en el proceso de evaluación, otorgando un papel más activo al alumnado a la vez que favoreciendo la comunicación y coordinación entre el profesorado.

El hecho de que la propuesta haya introducido novedades con respecto al desarrollo anterior de la asignatura, y estas hayan obtenido resultados satisfactorios con éxito, convierte a la experiencia realizada en una práctica que puede servir de inspiración a otros docentes universitarios y ser implementada en el futuro.

\section{Referencias bibliográficas}

Aubert, A., Flecha, A., García, C., Flecha, R., y Racionero, S. (2008). Aprendizaje dialógico en la sociedad de la información. Barcelona: Hipatia.

Akoschky, J., Alsina, P., Díaz, M., y Giráldez, A. (2008). La música en la Escuela Infantil. Barcelona: Graó.

Blández, J. (2010). La clase de educación física: escenario de la investigación. En C. González y T. Lleixà (Coords.) Educación Física. Investigación, innovación y buenas prácticas. Barcelona: Graó.

Botella Nicolás, A Mª., Adell Valero J. R. (2018). La integración de las artes a través de una propuesta didáctica en educación secundaria obligatoria: música, plástica y expresión corporal. Vivat Academia. Revista de Comunicación, 142, 109-123. doi http://doi. org/10.15178/va.2018.142.109-123

Calvo, A., y Susinos, T. (2010). Prácticas de investigación que escuchan la voz del alumnado: mejorar la Universidad indagando en la experiencia. Profesorado. Revista de currículum y formación del profesorado, 14(3), 75-88. Disponible en: http://www.ugr.es/local/ recfpro/rev143ART5.pdf

Carr, W., y Kemmis, S. (1988). Teoría crítica de la enseñanza. La investigación-acción en la formación del profesorado. Barcelona: Martínez Roca. 
Castillo-Cedeño, R., Ramírez-Abrahams, P., y RuízGuevara, L. S. (2017). Necesidades de formación profesional en el ámbito de la primera infancia: Percepción y aportes del estudiantado1. Revista electrónica educare, 21(1), 161-181. Doi: http://dx.doi.org/10.15359/ ree.21-1.9

Castro-Martínez, E. y Fernández de Lucio, I. (2013). El significado de innovar. Madrid: CSIC, Los libros de la Catarata.

Coll-Serrano, V., Pardo-García, C., y Pérez, P.J. (2018). Teaching-learning methods and their effect on professional development and the development of graduates' competencies. Culture and Education, 30(3), 556-583. Doi: https://doi.org/10.1080/11356405.2018.1494773

Coutinho, C. (2007). Cooperative learning in higher education using weblogs: a study with undergraduate students of education in Portugal. World Multiconference on Systemics, Cybernetic and Informatics, 11(1), 60-64. Consulta realizada 8 octubre. 2020. http://hdl. handle.net/1822/6721

Díaz, M., y Giráldez, A. (Coords.) (2013). Investigación cualitativa en educación musical. Barcelona: Graó.

Drent, M. y Meelissen, M. (2008). Which factors obstruct or stimulate teacher educators to use ICT innovatively? Computers y Education, 51, 1, 187-199. Doi: https://doi.org/10.1016/j.compedu.2007.05.001

Fullan, M. (2002). El significado del cambio educativo: un cuarto de siglo de aprendizaje. Profesorado, revista de currículum y formación del profesorado, 6 (1-2), $1-14$.

Gil, J., Rodríguez, G., y García, E. (1996). Metodología de la investigación cualitativa. Archidona, Málaga: Aljibe.

Iglesias-Cortizas, M.J., y Rodicio-García, M.L. (2013). El desarrollo de la creatividad e innovación. Un reto ante la crisis actual. Revista de Investigación en Educación, 11(1), 134-148.

Isusi-Fagoaga, R. (2018). Innovación, interdisciplina- riedad y educación artística en la formación docente universitaria. DEDiCA. Revista de Educação e Humanidades, 13, 43-53. Consulta realizada 8 octubre. 2020. http://hdl.handle.net/10481/50206

Khvilon, E. y Patru, M. (2004). Las tecnologías de la información y comunicación en la formación docente. París: UNESCO.

Kirk, D. (1990). Educación física y currículum: Introducción crítica. Valencia: Universitat de València.

Lavega, P., Sáez de Ocáriz, U., Lasierra, G., y Salas, C. (2013). Intradisciplinariedad e Interdisciplinariedad en la adquisición de competencias: estudio de una experiencia de aprendizaje cooperativo. Revista electrónica interuniversitaria de formación del profesorado, 16(1), 133-145. Doi: https://doi.org/10.6018/reifop.16.1.179491

Martínez, M. (2014). La investigación cualitativa (síntesis conceptual). Revista De Investigación En Psicología, 9(1), 123-146.

Martínez-Bello, V., y Bernabé-Villodre, M. D. M. (2019). Experiencia universitaria innovadora sobre didáctica de la motricidad infantil: De las aulas universitarias a las aulas de Educación Infantil. Revista Electrónica Educare, 23(3), 300-325. Doi: https://doi. org/10.15359/ree.23-3.15

Mínguez, X., Botella, A. M., Fernández, R., Martínez, S., y Angulo, T. (2014). Aprender de forma multidisciplinar en la universidad: una experiencia con alumnado de magisterio del grado de infantil. En Investigación e innovación en formación del profesorado (pp. 51-60). Murcia: Servicio de Publicaciones de la Universidad de Murcia.

Morales, P., y Landa, V. (2004). Aprendizaje basado en problemas. Theoria, 13, 145-157.

Pareja, J.A., y Pedrosa, B. (2014). Cambios en la metodología docente del espacio europeo de educación superior. Revista de Estudios y Experiencias en Educación, 13(26), 229-245. Disponible en: https://www. 
redalyc.org/articulo.oa? $\mathrm{id}=243132847013$

Rivera, E., y De la Torre, E. (2006). Democratizar el aula universitaria. Una propuesta alternativa de formación inicial universitaria desde la participación del alumnado. Investigación en la Escuela, 57, 85-96. Doi: https://doi.org/10.12795/IE.2005.i57.07

Rodríguez, G., Gil, J., y García, E. (1996). Metodología de la Investigación Cualitativa. Málaga: Algibe.

Sánchez-Valero, J.A., Arrazola-Carballo, J., y CalderónGarrido, D. (2017). The DIYLab project (do it yourself in education: Expanding digital competence to foster student agency and collaborative learning). Culture and Education, 29(4), 871-876. Doi: https://doi.org/10. $1080 / 11356405.2017 .1383637$

Schoepp, K. (2005). Barriers to Technology Integration in a Technology-Rich Environment. Learning and Teaching in Higher Education: Gulf Perspectives, Vol. 2, http://www.zu.ac.ae/lthe/vol2no1/lthe02_05.pdf

Summers, M., Childs, A., y Corney, G. (2005). Education for sustainable development in initial teacher training: issues for interdisciplinary collaboration. Environmental Education Research, 11(5), 623-647. Doi: https://doi.org/10.1080/13504620500169841

Vallés-Rapp, C., Gil-Puente, C., y López-Luengo, M.A. (2019). Una experiencia de evaluación formativa en el Máster de Formación del Profesorado de Educación Secundaria. Revista Infancia, Educación y Aprendizaje, 5(2), 246-250. Doi: https://doi.org/10.22370/ ieya.2019.5.2.1686

Vila, B., y Cardo, C. (2009). Material sensorial (0-3) años. Manipulación y experimentación. Barcelona: Graó. 